\title{
VALIDATION OF THE SPECTROPHOTOMETRIC PROCEDURE FOR DESLORATADINE ASSAY IN TABLETS APPLYING THE UNCERTAINTY CONCEPT OF THE STATE PHARMACOPOEIA OF UKRAINE
}

\author{
Dmytro Leontiev \\ Department of Pharmaceutical Chemistry ${ }^{l}$ \\ Department of Validation and Reference Standards ${ }^{2}$ \\ leontievd@yahoo.com \\ Vasyl Petrus \\ Department of Pharmaceutical Chemistry ${ }^{l}$ \\ petrus.vasyl@gmail.com \\ Natalia Volovyk \\ Department of Validation and Reference Standards ${ }^{2}$ \\ nvolovyk_rs@ukr.net \\ Oleksandr Gryzodub \\ Department of the State Pharmacopoeia of Ukraine ${ }^{2}$ \\ o.gryzodub@gmail.com \\ ${ }^{I}$ National University of Pharmacy \\ 53 Pushkinska srt., Kharkiv, Ukraine, 61002 \\ ${ }^{2}$ Ukrainian Scientific Pharmacopoeial Centre for Quality of Medicines \\ 33 Astronomichna str., Kharkiv, Ukraine, 61085
}

\begin{abstract}
Aim. This work aimed to validate an assay procedure for desloratadine tablets by direct spectrophotometric method.

Materials and methods. A pilot-scale batch of the pharmaceutical preparation Alerdez, film-coated tablets containing $5 \mathrm{mg}$ of desloratadine, manufactured by PJSC SIC «Borshchahivskiy CPP», Ukraine, was used as an object of the study. A UV-Vis spectrophotometer Lambda 25 (Perkin Elmer), analytical balance Mettler Toledo XP 205DR, and class A volumetric apparatus were used in the study. Validation of the procedure was performed following the metrological approach of the State Pharmacopoeia of Ukraine ( $\mathrm{SPhU}$ ), whose requirements for the target uncertainty and bias, which rest on the risk assessment of making incorrect decisions on compliance (a confidence level of $95 \%$ ), were translated into criteria for all validation characteristics recommended by ICH. All calculations were made in normalised coordinates. The linearity, accuracy and precision (repeatability) were studied in a single experiment using nine different concentrations that uniformly covered the range of $\pm 30 \%$ from the nominal concentration of desloratadine. For validation of the procedure, an SPhU reference standard of desloratadine was used.

Results. The experiment design and validation characteristics being tested were in full compliance with ICH Q2(R1) recommendations. All performance characteristics conformed to the criteria recommended by the SPhU. Requirements for the target uncertainty $(1.6 \%)$ and bias for any systematic source of variation $(\leq 0.51 \%$, negligible in relation to $1.6 \%)$ were established. The analytical procedure was specific - the absorbance from the placebo solution was insignificant (A $\%=0.36)$. The procedure met the requirements for linearity, accuracy, and precision at the repeatability level. The residual standard deviation $s_{0}$ was $0.34(\leq 0.84)$; correlation index $R_{c}$ was 0.9998 ( $\geq 0.9991$ ); intercept $a$ was 0.045 (less than its confidence interval $\Delta_{a}=1.14$ ). The confidence interval for recovery $\Delta_{Z}$, which was used as a precision estimate, was $0.55 \%$ (less than the target uncertainty). The mean recovery, which was used as an accuracy estimate, statistically insignificantly deviated from $100 \%\left(\left|Z_{\text {mean }}-100\right|=0.022 \%\right)$. The confidence interval for the intermediate precision $\Delta_{\text {intra }}$ was $0.33 \%$ (less than the target uncertainty). The developed analytical procedure was found to be robust.

Conclusions. A spectrophotometric procedure suitable for the assay of desloratadine in film-coated tablets Alerdez with content limits of $\pm 5 \%$ was validated by the SPhU approach.
\end{abstract}

Keywords: method validation, State Pharmacopoeia of Ukraine, target uncertainty, validation characteristics criteria, desloratadine tablets, assay. 


\section{Introduction}

Desloratadine (8-Chloro-6,11-dihydro-11-(piperidine-4-ylidene)-5H-benzo[5,6]cyclohepta[1,2-b]pyridine; CAS 100643-71-8) is used for the relief of symptoms of seasonal allergic rhinitis, perennial (non-seasonal) allergic rhinitis, and the symptomatic treatment of pruritus and urticaria (hives) associated with chronic idiopathic urticarial, usually in the pharmaceutical formulations of tablets, syrups and solutions [1].

Desloratadine can be easily determined by the direct absorption spectrophotometry (SP) in the UV region due to the strong absorption of desloratadine solutions [2]. The substance of desloratadine is described in the European Pharmacopoeia (Ph. Eur.) and the United States Pharmacopeia (USP). The USP also has monographs «Desloratadine Tablets» and «Desloratadine Orally Disintegrating Tablets». The method of liquid chromatography with UV detector is used for desloratadine assay in all abovementioned monographs. It has potential advantages since, as a rule, it is a stability-indicating method. On the other hand, the SP method of desloratadine assay has an asset of being a faster method, which is especially valuable for pharmaceutical companies for both in-processing control and batch release.

An analytical procedure must be validated before its implementation into routine use. The methodology of the analytical procedure validation described in the ICH Guide Q2(R1) «Validation of analytical procedures: text and methodology» is generally accepted [3]. The aim of the validation is to demonstrate the suitability of the procedure for its intended use. However, ICH Q2(R1) and other regulatory documents (namely, recommendations of the EDQM [4], USP [5], and WHO [6], which, despite having a different content, follow the ICH guide) do not specify criteria for demonstrating such suitability.

The concept of the uncertainty of measurement result is generally recognised as a scientific basis for applied metrology. The importance of the uncertainty concept for the pharmaceutical sector is emphasised in scientific articles, e. g. in work [7]. Recently, the USP Expert Panel has proposed a life cycle approach to validation that is based on the uncertainty concept [8] but does not follow the ICH recommendations. Similarly, based on the uncertainty approach [9], the new concept of Analytical Quality by Design provides only general principles for quality assurance. It should be noted that the uncertainty concept is now widely applied in analytical chemistry [10]. It takes into account the risk of making an incorrect conclusion on specification compliance at the accepted level of reliability [11]. The uncertainty concept can be correctly implemented in the pharmaceutical sector only if internationally accepted standardisation rules are considered [12]. The decision rules take account of the fact that all sources of variability associated with the analytical result, technology, stability changes, etc., are included in the specifications. As a result, a decision on compliance is taken simply on the basis whether an analytical result meets specifications. The uncertainty approach can be correctly used for such a straightforward decision rule providing that variability of the analytical assay result for the finished preparation is insignificant in comparison with the two-sided specification width [12]. Based on these principles, the State Pharmacopoeia of Ukraine (SPhU) established requirements for the target uncertainty for main pharmaceutical tests that depend on the specification width and vary for assays of finished drug products and substances [13]. After public discussion in the journal «Farmacom» [14], an approach to the analytical procedure validation was introduced in the State Pharmacopoeia of Ukraine [15]. In fact, the SPhU approach formulated the Target Analytical Profile for the analytical procedure, which was later proposed by the USP as part of the novel concept of Lifecycle Management of Analytical Procedures [8], which was then issued as a draft to the USP monograph [16]. The life cycle approach states that $\mathrm{ICH}$ validation characteristics are important and necessary to meet the required level of quality of the data [17] but do not provide recommendations for validation criteria. In contrast, the SPhU approach translates requirements for the target uncertainty and bias into criteria for all performance characteristics recommended in Q2(R1).

Although there is a number of papers dedicated to the development and validation of the SP assay of desloratadine in tablets, we met only one that employed the uncertainty concept [18], but only to estimate the uncertainty of measurement for the procedure being developed rather than to demonstrate the suitability of the procedure for the intended use by comparison of the validation 
results with criteria set in advance. Therefore, it is topical to assess the suitability of the analytical procedure intended for SP assay of desloratadine by the SPhU approach, i.e. based on the measurement uncertainty concept and risk assessment of making a wrong decision about the compliance with specifications.

The objective of this work is to validate a procedure for assay of desloratadine in film-coated tablets (tablet strength $5 \mathrm{mg}$, content limits of desloratadine $\pm 5 \%$ ) produced by PJSC SIC «Borshchahivskiy CPP», Ukraine, following the requirements of the SPhU.

\section{Materials and methods}

\subsection{Test object}

A pilot-scale batch of Alerdez (Lot No. E01), $5 \mathrm{mg}$ desloratadine film-coated tablets produced by «Borshchahivskiy CPP», Ukraine, served as an object of the study. The content of desloratadine per tablet is specified $5 \mathrm{mg} \pm 5 \%$ (from $4.75 \mathrm{mg}$ to $5.25 \mathrm{mg}$ ). The weight of one tablet is about $105 \mathrm{mg}$.

Excipients: calcium hydrogen phosphate dihydrate, cellulose, maise starch, talc, hypromellose, macrogol 400 (PEG 400), macrogol 4000 (PEG 4000), polyvinyl alcohol, titanium dioxide, indigo-carmine colouring.

\subsection{Reference standards and reagents}

A desloratadine reference standard of the State Pharmacopoeia of Ukraine suitable for SP assay was used (the assigned value: $99.7 \%$; target uncertainty: $0.5 \%$, expressed as a one-sided confidence interval for a reliability level of $95 \%$ ).

All reagents used in the study met the requirements of the $\mathrm{Ph}$. Eur.

The desloratadine stock solution $(1 \mathrm{mg} / \mathrm{mL})$ was prepared for the linearity and stability studies. It was also used for the preparation of model solutions by the gravimetric method.

\subsection{Equipment}

The spectrophotometers Lambda 25 and Lambda $35^{*}$ equipped with a 1 -cm cuvette (Perkin Elmer), analytical balances Mettler Toledo XP 205DR and Kern ABS 220-4*, pH-meter Metrohm, Class A volumetric pipettes and flasks, PTFE filter with pre-filter $0.45 \mu \mathrm{m}$ cat. No. SYTG0602MNXX104 manufactured by MDI were used in the study.

*Note: these were used additionally in the inter-laboratory precision experiment.

\section{Results}

\subsection{An analytical procedure being validated}

The text of the analytical procedure is given below.

Test solution. Grind 20 tablets to a visually homogeneous mass. Dilute $105 \mathrm{mg}$ of the obtained sample in $250 \mathrm{~mL}$ of 0.1 M hydrochloric acid, keeping it in an ultrasonic bath for 15 minutes, and filter.

Reference solution. Dissolve $40 \mathrm{mg}$ of the desloratadine reference standard in $100 \mathrm{~mL}$ of $0.1 \mathrm{M}$ hydrochloric acid. Dilute $5.0 \mathrm{~mL}$ of the obtained solution in $100 \mathrm{~mL}$ of 0.1 M hydrochloric acid.

Measure the absorbance at a wavelength of $282 \mathrm{~nm}$.

The length of the optical path: $10 \mathrm{~mm}$.

The compensation solution: 0.1 M hydrochloric acid.

The content of desloratadine $\left(X_{2}\right)$ per tablet (in $\mathrm{mg}$ ) is calculated by the formula:

$$
X_{2}=\frac{A_{1} \times m_{0} \times P \times b \times 5 \times 250}{A_{0} \times m_{1} \times 100 \times 100 \times 100}=\frac{A_{1} \times m_{0} \times P \times b}{A_{0} \times m_{1} \times 800},
$$

where $A_{1}$ - absorbance of the test solution; $A_{0}$ - absorbance of the reference solution; $m_{0}-$ test portion of the desloratadine reference standard, in $\mathrm{mg} ; m_{1}$ - test portion of the sample obtained by tablet grinding, in $\mathrm{mg} ; P$ - assigned value of desloratadine in the reference standard, in per cent; $b$ - average mass of tablets, in $\mathrm{mg}$.

For the nominal concentration of desloratadine $(0.02 \mathrm{mg} / \mathrm{mL})$, the absorbance is about 0.64 absorption units (AU). 


\subsection{Design of the validation experiment and criteria}

The detailed explanation of the theoretical principles of the SPhU approach is given in the monograph [19].

\subsubsection{The target uncertainty of analytical results}

Under the SPhU approach [11], the target uncertainty for assays of finished drug products $\left(\max \Delta_{A s}\right)$ should be insignificant compared to the width of two-sided specifications:

$$
\max \Delta_{A s}=0.32 \times B,
$$

where $B$ - half-width of content limits; $\max \Delta_{A s}$ - target uncertainty.

Hereafter under an expanded uncertainty, we understand a $95 \%$ one-sided confidence interval.

The coefficient of 0.32 provides a $95 \%$ reliability of making a correct decision on compliance $[13,19]$.

Then, for desloratadine tablets as a finished drug product with the content limits of $\pm 5 \%$, the requirement for insignificance takes the following form:

$$
\max \Delta_{A s}=1.6 \%
$$

\subsubsection{Use of normalised coordinates}

When using normalised coordinates, we assume that during routine analysis, a single reference solution is used for calibration, i. e. the calibration curve passes through the origin.

The normalised coordinates are defined as follows:

$$
X_{i}=\frac{C_{i}}{C^{s t}} \times 100 \%, \quad Y_{i}=\frac{A_{i}}{A^{s t}} \times 100 \%, \quad Z_{i}=\frac{Y_{i}}{X_{i}} \times 100 \%,
$$

where $C_{i}$ - concentration of the analyte in the $i$-th model solution being analysed; $C_{s t}-$ concentration of the analyte in the reference solution; $A_{i}$ - analytical signal of the analyte for the $i$-th model solution being analysed; $A_{s t}$ - analytical signal of the analyte for the reference solution.

The concentration of the analyte in the reference solution should be close to the nominal concentration.

In normalised coordinates, for the calibration curve that passes through the origin, $Z$ is the recovery expressed in per cent.

All further calculations are carried out in the normalised coordinates.

\subsubsection{Criteria for performance characteristics}

\section{Specificity}

According to the SPhU approach, bias caused by the additional absorbance of the placebo components $\left(\delta_{\text {placebo }}\right)$ should be insignificant in relation to $\max \Delta_{A s}$ :

$$
\delta_{\text {placebo }}, \% \leq 0.32 \times \max \Delta_{A s} .
$$
as follows:

For content limits of $\pm 5 \%$, the requirement for the insignificance of $\delta_{\text {placebo }}$ is expressed

$$
\delta_{\text {placebo }} \leq 0.51
$$

\subsubsection{Range}

By the $\mathrm{ICH}$ recommendations [3], the range of the linearity study in coordinates $\left(X_{i}, Y_{i}\right)$ should be not less than $80-120 \%$ for assay and not less than $70-130 \%$ for the content uniformity.

Since the procedure is intended to be used for the assay and content uniformity, the range of $70-130 \%$ and the most stringent acceptance criteria as for the assay with content limits of $\pm 5 \%\left(\max \Delta_{A S}=1.6 \%\right)$ are applied. 


\subsubsection{Linearity, accuracy, precision (repeatability)}

The linearity, accuracy, and precision (at the repeatability level) are studied simultaneously for nine concentrations, evenly distributed across the range, which complies with the ICH recommendations for precision at the repeatability level. Actual concentrations that were studied are given in Table 1 in normalised coordinates.

\section{Linearity - the requirement for the residual standard deviation $s_{0}$}

The residual standard deviation $s_{0}$ characterises a dispersion of the points around the line. Since each point can be treated as an individual analytical result, $s_{0}$ in the normalised coordinates is $R S D$ for the standard uncertainty of analytical results. Therefore, the confidence interval calculated from $s_{O}$ should not exceed $\max \Delta_{A s}$, i. e.:

$$
t(95 \%, g-2) \times s_{0} \leq \max \Delta_{A s},
$$

where $g$ is the number of model solutions.

For $g=9$ and content limits of $\pm 5 \%$, the requirement takes the following form:

$$
s_{0} \leq 1.6 / 1.8946 \leq 0.84 \text {. }
$$

Linearity - the requirement for the correlation index $R_{c}$

For the estimation of validation results, the correlation index $\left(R_{c}\right)$ is used following the SPhU approach:

$$
\begin{gathered}
R_{c}=\sqrt{1-\frac{s_{0}^{2}}{S D_{\text {range }}^{2}},} \\
S D_{\text {range }}=\sqrt{\frac{\sum_{i=1}^{g}\left(X_{i}-\bar{X}\right)^{2}}{g-1}},
\end{gathered}
$$

where $X_{i}$ - concentration of the $i$-th model solution used for the linearity study, in per cent of the nominal concentration; $g$ - number of the model solutions.

Due to the relative complexity of the mathematical expression of the Pearson coefficient, it is difficult to formulate the requirements connecting the values of $r$ with the requirements for the uncertainty of the analysis results [20]. Yet it is easy to develop requirements for $R_{c}$ based on those for $\max \Delta_{A s}$. Unlike the Pearson coefficient, which is only applicable to the linear regression, $R_{c}$ can be used for any regression.

Taking into account the requirements for $s_{0}$, the requirements for $R_{c}$ can be expressed as follows:

$$
R_{c} \leq \sqrt{1-\frac{0.84^{2}}{S D_{\text {range }}^{2}}} .
$$

$S D_{\text {range }}$ is calculated from the actual concentrations of model solutions.

\section{Linearity - the requirement for the intercept (a)}

For the intercept (a), a two-level criterion is used.

The statistically insignificant difference from zero: the value of $a$ should be less than its confidence interval.

Therefore, for $g=9$, statistical insignificance can be expressed as follows:

$$
a \leq t(95 \%, g-2) \times s_{0} ; a \leq 18946 \times s_{0},
$$

where $s_{a}$ is the standard deviation of the intercept of the line $(a)$ obtained by the least-squares method. 
The practically insignificant difference from zero: the value of $a$ should be practically insignificant for the intended use of the procedure if the bias due to non-passage of the calibration curve through the origin does not exceed $0.32 \times \max \Delta_{A s}$.

For the symmetric range of application of the procedure, the criterion of practical insignificance takes the following form:

$$
a \leq\left|\frac{0.32 \times \Delta_{A s}}{1-\left(X_{\min } / 100\right)}\right|,
$$

where $X_{\min }$ is the minimum limit of the procedure application range.

The criterion of practical insignificance (11) is only used in the event when the criterion of statistical insignificance (10) is not met.

For the content limits of $\pm 5 \%$ and the range of $70-130 \%$, the requirement takes the following form:

$$
a \leq 1.71 \text {. }
$$

Precision - estimation from the linearity data

For the estimation of the procedure precision from the linearity study, $Z_{i}$ values are used:

$$
\Delta_{Z}=t(95 \%, g-1) \times S D_{Z}=1.8595 \times S D_{Z}
$$

The following relationship must be fulfilled:

$$
\Delta_{Z} \leq \max \Delta_{A s}
$$

For content limits of $\pm 5 \%$, the requirement takes the following form:

$$
\Delta_{Z} \leq 1.6 \%
$$

\section{Accuracy - estimation from the linearity data}

Bias $(\delta)$ of the analytical results is defined as follows:

$$
\delta \%=|\bar{Z}-100|
$$

By the SPhU approach, bias should be insignificant for spectrophotometric assay.

For accuracy estimation, a two-level criterion is used.

Statistical insignificance: the value of $\delta$ should be statistically insignificantly different from zero:

$$
\delta \leq \frac{\Delta_{\bar{Z}}}{\sqrt{g}},
$$

where $g$ is the number of solutions used for the accuracy study from the linearity data.

For $g=9$ and content limits of $\pm 5 \%$, the following requirement for bias should be met:

$$
\delta \leq \Delta_{Z} / 3
$$

Practical insignificance: if the failure to comply with the requirements for statistical insignificance is observed, the practical insignificance $\delta$ should be checked:

$$
\delta \leq 0.32 \times \max \Delta_{A s} .
$$

For content limits of $\pm 5 \%$, the requirement for the practical insignificance is expressed as follows:

$$
\delta \leq 0.51
$$




\subsubsection{Intermediate precision}

For the intermediate precision, we use the approach based on the SPhU concept, which is described in detail in our paper [15]. Analyse the same sample of ground tablets on $m \geq 2$ different days. Prepare $n \geq 5$ of independent test solutions each day, and analyse them varying the factors that could affect precision as much as possible (different analysts, equipment, etc.). If all factors affecting precision are insignificant, all the results must belong to the same population. Therefore, for all obtained assay results $\left(Z_{i}\right)$, the mean $\left(Z_{\text {intra }}\right)$, standard deviation $\left(S D_{\text {Zintra }} \%\right)$, and relative confidence interval $\left(\Delta_{\text {intra }} \%\right)$ are calculated:

$$
\begin{gathered}
\Delta_{\text {intra }}=t[95 \%, n \times(m-1)] \times S D_{Z_{\text {intra }}}, \\
\Delta_{\text {intra }} \text { should not exceed } \Delta_{A s}, \\
\Delta_{\text {intra }} \leq \max \Delta_{A s} .
\end{gathered}
$$

For content limits of $\pm 5 \%$, the requirement (25) takes the following form:

$$
\Delta_{\text {intra }} \leq 1.6 \% \text {. }
$$

\subsubsection{Robustness - stability of solutions}

Based on the SPhU concept, we propose the following approach to stability studying.

Prepare a test solution according to the procedure. Prepare a reference solution with precisely known concentrations of analyte («zero» solutions). Use the gravimetric method for dilutions. Assay the solutions («zero» time). After a predetermined period (we propose to check stability in $N=5 \mathrm{~h}, 24 \mathrm{~h}$ and $48 \mathrm{~h}$ after «zero» solutions preparation), prepare a fresh reference solution in the same manner ( $N$-th solution). At the specified time, analyse both of the «zero» solutions using a fresh reference solution. Express results in per cents in relation to «added» amount for «zero» reference solution and in relation to «found» amount for «zero» test solution $\left(Z_{N}\right)$; the desloratadine content in «zero» solutions is taken as $100 \%$. Calculate differences in concentrations $\left(100 \%-Z_{N}\right)$. The solutions are stable as long as the difference is insignificant in relation to $\max \Delta_{A s}$ :

$$
\left|100 \%-Z_{N}\right| \leq 0.32 \times \max \Delta_{A s} .
$$

Since the specific absorbance of degradation products may differ from one of the analytes upward and downward, the difference modulus is used.

For content limits of $\pm 5 \%$, the requirement (26) is expressed as follows:

$$
\Delta_{\text {Stab }}(\%) \leq 0.51
$$

This approach allows us to confirm the stability of the solutions for quite a long period of time, which may be critical for a laboratory when investigating the OOS situation. However, the necessity to prepare solutions with the precisely known concentrations may be seen as a disadvantage.

\subsubsection{Robustness - the reliability of the analysis with respect to deliberate variations} in method parameters

The solubility of desloratadine is increased in acidic water solutions in comparison with pure water. The UV spectrum of desloratadine is also affected by $\mathrm{pH}$ of water diluent [18]. Consequently, it is reasonable to investigate the impact of $\mathrm{HCl}$ concentration variation in the diluent on the reference solution extinction and desloratadine recovery from the test portion of ground tablets.

For the prognosis of the "worst case» of $0.1 \mathrm{~N} \cdot \mathrm{HCL}$ concentration variability in the routine analysis, the uncertainty of the laboratory procedure of $0.1 \mathrm{~N} \cdot \mathrm{HCl}$ preparation was estimated per the $\mathrm{SPhU}$ recommendations. It comprised of about $2 \%$. On that basis, according to the SPhU insignificance principle, an analytical procedure will be robust if the influence of $\mathrm{HCl}$ concentration 
variability in the diluent on the reference solution absorbance is still insignificant for the range of $\mathrm{HCl}$ concentration of $\pm 2 \% \times 3.2= \pm 7 \%$ from the nominal concentration of $0.1 \mathrm{~N} \cdot \mathrm{HCl}$. Following the same principle, the impact of $\mathrm{HCl}$ concentration variability is insignificant if the absorbance for reference solutions prepared using $0.093 \mathrm{~N} \cdot \mathrm{HCl}$ and $0.107 \mathrm{~N} \cdot \mathrm{HCl}$ does not differ from the absorbance of the reference solution prepared using $0.1 \mathrm{~N} \cdot \mathrm{HCl}$ by more than $0.51 \%$. In the same way, the variability of $\mathrm{HCl}$ concentration in the diluent has an insignificant impact on the desloratadine recovery if the «found» value for any test solutions prepared using $0.093 \mathrm{~N} \cdot \mathrm{HCl}, 0.1 \mathrm{~N} \cdot \mathrm{HCl}$ and $0.107 \mathrm{~N} \cdot \mathrm{HCl}$ does not deflect by more than 0.51 from the «true» value that should be obtained with the nominal $\mathrm{HCl}$ concentration. It means that the difference between «found» values for any two solutions must not exceed $0.51 \% \times \sqrt{2}=0.74 \%$, because both experimental values vary independently and can have uncertainty that should not exceed 0.51 .

\subsection{Experimental results}

UV spectra of the reference solution, test solution, model solution, and placebo solution are given in Fig. 1.
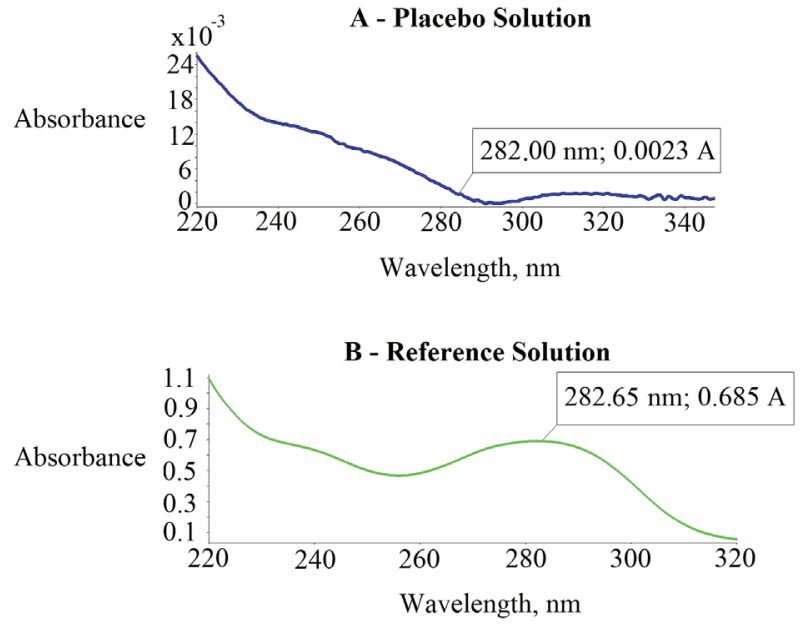

C - Test Solution
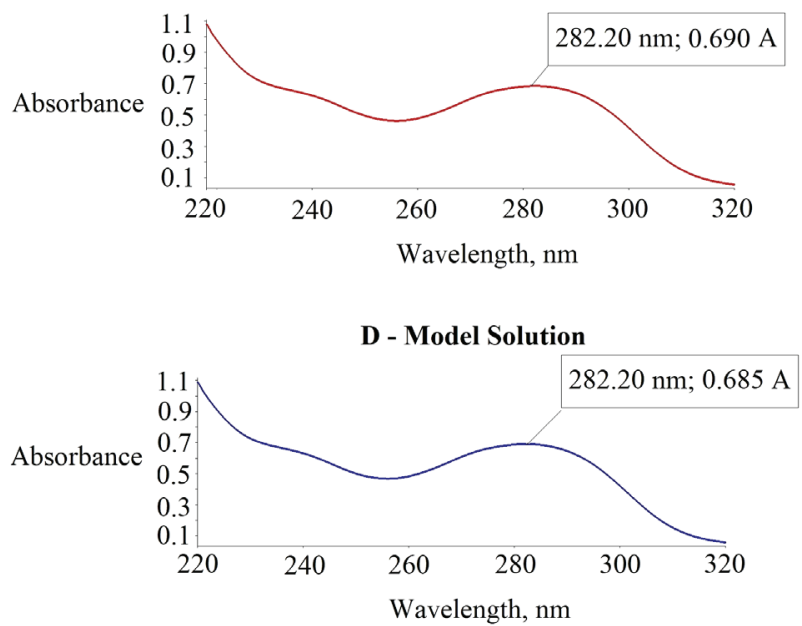

Fig. 1. Specificity study: the UV spectrum of (A) Placebo Solution, (B) Desloratadine Reference Solution, (C) Test Solution, (D) Model Solution.

\subsubsection{Specificity}

The test solution prepared from the ground tablets for the stability evaluation, the «100\%» model solution prepared for the linearity evaluation and the reference solution showed identical spectra. 
The extinction of this solution was recalculated per the nominal concentration of the desloratadine as follows:

$$
A_{\text {Nom }}=A_{R e f} \times C_{\text {Nom }} / C_{R e f}
$$

Then two solutions of placebo were prepared and measured following the procedure. The results of the specificity evaluation are shown in Table 1.

Table 1

Demonstration of specificity - the influence of the placebo absorption

\begin{tabular}{|c|c|c|c|}
\hline \multicolumn{4}{|c|}{ Recalculation of the extinction for the reference solution } \\
\hline \multicolumn{2}{|c|}{ Reference solution } & \multicolumn{2}{|c|}{$A_{\text {Nom }}(282 \mathrm{~nm}), C_{\text {Nom }}=0.020 \mathrm{mg} / \mathrm{mL}$} \\
\hline$A_{\text {Ref }}(282 \mathrm{~nm})$ & 0.685 & \multirow{2}{*}{\multicolumn{2}{|c|}{0.6447}} \\
\hline$C_{R e f}, \mathrm{mg} / \mathrm{mL}$ & 0.02125 & & \\
\hline \multicolumn{4}{|c|}{ Evaluation of the placebo influence } \\
\hline & $A, 282 \mathrm{~nm}$ & $A, 282 \mathrm{~nm}$, mean & Placebo influence, $\%\left(\delta_{\text {placebo }}\right)$ \\
\hline Placebo solution 1 & 0.0023 & \multirow{2}{*}{0.0023} & \multirow[b]{2}{*}{0.36} \\
\hline Placebo solution 2 & 0.0023 & & \\
\hline \multirow[t]{2}{*}{ Reference solution $100 \%$} & 0.6447 & - & \\
\hline & nsignificance & & $\leq 0.51 \%$ \\
\hline
\end{tabular}

The validation requirements for the specificity of the procedure are met.

\subsubsection{Linearity. Accuracy. Precision}

Results of the analysis of model solutions are given in Table 2.

Table 2

Results of linearity, accuracy, and precision

\begin{tabular}{cccccc}
\hline Solutions & Concentration, $\mathbf{m g} / \mathbf{m L}$ & $\boldsymbol{A}$, mean for 3 measurements & Amount added $\left(\boldsymbol{X}_{\boldsymbol{i}}\right)$ & Amount found $\left(\boldsymbol{Y}_{\boldsymbol{i}}\right)$ & Recovery $\left(\boldsymbol{Z}_{\boldsymbol{i}}\right)$ \\
\hline Test 1 & 0.014076 & 0.455 & 66.24 & 66.42 & 99.86 \\
Test 2 & 0.015999 & 0.515 & 75.29 & 75.18 & 99.89 \\
Test 3 & 0.016925 & 0.545 & 79.65 & 79.56 & 100.09 \\
Test 4 & 0.018039 & 0.582 & 84.89 & 84.96 & 100.05 \\
Test 5 & 0.020143 & 0.650 & 94.79 & 94.84 & 99.94 \\
Test 6 & 0.021771 & 0.701 & 102.45 & 102.38 & 100.46 \\
Test 7 & 0.023294 & 0.754 & 109.62 & 110.12 & 99.42 \\
Test 8 & 0.024296 & 0.779 & 114.33 & 113.67 & 100.21 \\
Test 9 & 0.026271 & 0.849 & 123.63 & 123.89 & 100.28
\end{tabular}

For the obtained results, the parameters of the linear relationship were calculated by the least-squares method:

$$
Y_{i}=a+b \times X_{i}
$$

where $Y_{i}$ - concentrations of the model solutions, in normalised coordinates; $X_{i}$-absorbance of the model solutions, in normalised coordinates; $a$-intercept; $b$-slope.

The graph of the linear relationship is shown in Fig. 2. 


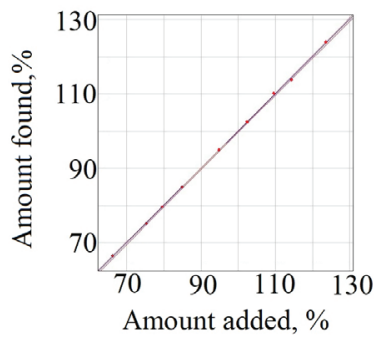

Fig. 2. The linear relationship between normalised concentrations $(X)$ and normalised absorbance $(Y)$ for desloratadine model solutions from the linearity study

\section{Linearity}

Results of the calculation of parameters of the linear relationship between normalised concentrations $(X)$ and normalised absorbance $(Y)$ for model solutions of desloratadine and their assessment are shown in Table 3.

Table 3

Results of the linearity study

\begin{tabular}{|c|c|c|c|c|}
\hline Parameters & Values & $\begin{array}{l}\text { Criterion 1: Requirement of } \\
\text { statistical insignificance }\end{array}$ & $\begin{array}{l}\text { Criterion 2: Requirement of } \\
\text { practical insignificance }\end{array}$ & Conclusion \\
\hline$b$ & 0.9997 & & & \\
\hline$a$ & $|0.045|$ & $\leq 1.14$ & $\leq 1.71$ & Satisfies criterion 1 \\
\hline$s_{a}$ & 0.60 & & & \\
\hline$s_{0}$ & 0.34 & $\leq 0.84$ & & Satisfies \\
\hline$S D_{\text {range }}$ & 20.16 & & & \\
\hline$R_{C}$ & 0.9998 & $\geq 0.9991$ & & Satisfies \\
\hline
\end{tabular}

The validation requirements for the linearity of the procedure are met.

Precision and accuracy

The assessment results of the procedure precision and accuracy from the linearity data are shown in Table 4.

Table 4

Results of the study of accuracy and precision

\begin{tabular}{cccccc}
\hline & Parameters & Values & $\begin{array}{c}\text { Criterion 1: Requirement of } \\
\text { statistical insignificance }\end{array}$ & $\begin{array}{c}\text { Criterion 2: Requirement } \\
\text { of practical insignificance }\end{array}$ & Conclusion \\
\hline Precision & $\Delta_{Z}$ & 0.55 & $\leq 1.6$ & $\leq 0.51$ & Satisfies \\
Accuracy & $\left|Z_{\text {mean }}-100\right|$ & $|0.022|$ & $\leq 0.185$ & Satisfies criterion 1
\end{tabular}

The validation requirements for precision and accuracy of the procedure are met.

\subsubsection{Intermediate precision}

Test solutions were prepared by the procedure described in Section 2 Materials and methods. Test portions for the assay on the $1^{\text {st }}$ and $2^{\text {nd }}$ days were taken from the same sample of ground tablets. Different analysts conducted an analysis on the $1^{\text {st }}$ and $2^{\text {nd }}$ day, using different reagents, volumetric glassware, filters, balances, ultrasonic baths, and spectrophotometers.

The results of the intermediate precision study are shown in Table 5. 
Table 5

Results of the intermediate precision study

\begin{tabular}{ccc}
\hline \multicolumn{3}{c}{ Desloratadine content found, mg } \\
\hline & Day 1 & Day 2 \\
100.84 & & 100.74 \\
100.53 & 100.41 \\
100.76 & 100.51 \\
100.78 & & 100.41 \\
100.80 & & 100.40 \\
100.85 & & 100.47 \\
$Z_{\text {intra }}=100.6$ & $S D_{\text {Zintra }}=0.184 \quad$ Criterion: $\Delta_{\text {intra }} \leq 1.6 \%$
\end{tabular}

The validation requirements for the intermediate precision of the analytical procedure are met.

\subsubsection{Robustness - stability of the solutions}

For the preparation of the test solution, the ground tablets of desloratadine were used as described in Section 3.3.3 Intermediate precision.

The solutions were stored at $20^{\circ} \mathrm{C}$, protected from direct sunlight.

The results of the stability study of the solutions are shown in Table 6.

Table 6

Results of the stability study

\begin{tabular}{ccccc}
\hline \multirow{2}{*}{ Solution } & Parameter & \multicolumn{3}{c}{ Time $(\boldsymbol{N}), \mathbf{h}$} \\
\cline { 3 - 5 } & & $\mathbf{5}$ & $\mathbf{2 4}$ & $\mathbf{4 8}$ \\
\hline Reference solution & $100 \%-Z$ & 100.09 & 99.74 & 100.11 \\
\multirow{2}{*}{ Test solution } & $Z, \%$ & -0.09 & 0.26 & -0.11 \\
& $100 \%-Z$ & 100.14 & 99.99 & 0.009 \\
& Criterion of insignificance: $|100-Z| \leq 0.51 \%$ & & -0.16
\end{tabular}

Validation requirements for stability of the solutions are met when analysed in $24 \mathrm{~h}$.

\subsubsection{Robustness - reliability of the analysis with respect to deliberate variations in the method parameters}

The stock solution of desloratadine with concentration about $50 \mathrm{mg} / 50 \mathrm{~mL}$ in $0.1 \mathrm{~N} \cdot \mathrm{HCl}$ was prepared. The diluents with $\mathrm{HCl}$ normality of 0.093 and 0.107 were prepared. The reference solutions for the evaluation of the impact of $\mathrm{HCl}$ concentration in the diluent on the reference solution extinction were prepared as follows: $5.0 \mathrm{~mL}$ (weighed) of the stock solution were diluted to $250 \mathrm{~mL}$ using $0.093 \mathrm{~N} \cdot \mathrm{HCl}, 0.107 \mathrm{~N} \cdot \mathrm{HCl}$, and $0.1 \mathrm{~N} \cdot \mathrm{HCl}$ as diluents. The absorbance of the prepared solutions was measured as described in the analytical procedure. Then, the absorbance of the reference solutions that were prepared using $0.093 \mathrm{~N} \cdot \mathrm{HCl}$ and $0.107 \mathrm{~N} \cdot \mathrm{HCl}\left(A_{\text {init }}\right)$ were recalculated per the concentration of reference solution with the nominal concentration of $\mathrm{HCl}\left(A_{n o m}\right)$ by the formula:

$$
A_{\text {recalc }}=A_{\text {init }} \times m_{\text {nom }} / m_{\text {init }},
$$

where $m$ - weights of aliquots of the reference solutions. 
The results of the impact of the diluent normality on the desloratadine spectra are shown in Table 7.

Table 7

Recalculated absorbance of reference solutions for the assessment of the impact of $\mathrm{HCl}$ normality in the diluent on the desloratadine spectrum

\begin{tabular}{ccc}
\hline Solution & Absorbance, A & Difference, \% \\
\hline Reference solution $(0.1 \mathrm{~N} \mathrm{HCl})$ & 0.640 & - \\
Reference solution $(0.093 \mathrm{~N} \mathrm{HCl})$ & 0.641 & $0.16 \%$ \\
Reference solution $(0.107 \mathrm{~N} \mathrm{HCl})$ & 0.640 & $0 \%$ \\
Criterion & & Difference, $\% \leq 0.51$
\end{tabular}

Three test solutions were prepared using $0.093 \mathrm{~N} \cdot \mathrm{HCl}, 0.1 \mathrm{~N} \cdot \mathrm{HCl}$, and $0.107 \mathrm{~N} \cdot \mathrm{HCl}$ as a diluent. The ratios of absorbance to the test portions were calculated for each test solution $(\mathrm{A} / \mathrm{m})$. As was substantiated above, the obtained values for the test solutions prepared using $0.093 \mathrm{~N} \cdot \mathrm{HCl}$, and $0.107 \mathrm{~N} \cdot \mathrm{HCl}$ must not deviate from the value obtained for test solution prepared using $0.1 \mathrm{HCl}$ by more than $0.74 \%$. The results are shown in Table 8 .

\section{Table 8}

Ratios of absorbance to the test portions for test solutions for the assessment of the impact of $\mathrm{HCl}$ normality in the diluent on the recovery of desloratadine from ground tablets

\begin{tabular}{ccccc}
\hline Diluent & Sample portion, $\mathbf{m}(\mathbf{m g})$ & Absorbance of the test solution, A & Ratio A/m & Difference, $\%$ \\
\hline $0.1 \mathrm{~N} \cdot \mathrm{HCl}$ & 410.5 & 0.6175 & 0.00150 & - \\
$0.093 \mathrm{~N} \cdot \mathrm{HCl}$ & 400.6 & 0.6003 & 0.00150 & $0 \%$ \\
$0.107 \mathrm{~N} \cdot \mathrm{HCl}$ & 415.2 & 0.6251 & 0.00151 & $0.67 \%$
\end{tabular}

Criterion

Difference, $\% \leq 0.72$

Evidently, the analytical procedure is robust regarding the deliberate variation of $\mathrm{HCl}$ concentration in the diluent.

\section{Discussion}

The validation results show that the analytical procedure is suitable for the intended use by the SPhU metrological criteria.

It should be noted that even though the $\mathrm{SPhU}$ metrological criteria were developed based on the ICH recommendations, the ICH approach itself does not allow making a conclusion about the suitability of the analytical procedure as it does not specify what the suitability of the analytical procedure for the use for a given application is. Therefore, from our point of view, all validation studies that are based only on ICH recommendations list the research results without the scientifically sound justification of suitability. The concept of uncertainty made it possible to formulate an idea of demonstrating the fitness of the procedure for its intended purpose, based on which the SPhU translated all ICH recommendations into criteria thus providing scientific justification to that idea.

Based on the uncertainty concept, the life cycle approach proposed later by the USP recognised the importance of ICH recommendations but did not translate them into criteria for ICH validation characteristics [17]. As a result, this approach is still separated from the internationally accepted ICH recommendations and even has some contradictions with them.

On the other hand, the USP approach has strongly criticised the one of ICH for being a formal check-box exercise that does not bear in mind the risk of incorrect decision-making in the 
routine application of the analytical procedure and not leading to the reliable understanding and control of the variability sources $[8,17]$, which, in fact, is also applicable to the SPhU.

In contrast to the ICH approach, USP proposed to use powerful tools for the quality assurance of the analysis result developed for the control of manufacture process (Lifecycle, Target Profile, Risk Management, Control Strategy, Knowledge Management, and Quality by Design) that enormously strengthen the effectiveness of the analytical validation process.

Study limitations. The validation experiment design was developed by the SPhU approach for pharmaceutical preparation «Alerdez», $5 \mathrm{mg}$ desloratadine film-coated tablets produced by «Borshchahivskiy CPP», Ukraine, and might not be suitable for validation of analytical procedures for desloratadine assay in film-coated tablets of other composition and/or manufacture.

The prospects for the further research. Considering those above, further, it seems reasonable to combine the SPhU metrological approach with the USP recommendations for quality assurance of analytical results.

\section{Conclusions}

1. The procedure for assay of desloratadine in film-coated tablets «Alerdez» with content limits of $\pm 5 \%$ manufactured by PJSC SIC «Borshchahivskiy CPP», Ukraine, by absorption spectrophotometry in the UV region was validated following the recommendations of the State Pharmacopoeia of Ukraine.

2. All validation characteristics and associated performance characteristics were examined in full compliance with ICH recommendations. The analytical procedure has been shown to be suitable for its intended use.

3. The uncertainty of the analytical results and bias ensure acceptable reliability of the decision on medicine compliance with specifications.

\section{Conflict of interests}

The authors declare that they have no conflicts of interest.

\section{References}

[1] Desloratadine (2020). National Center for Biotechnology Information PubChem. Compound Summary for CID 124087. Available at: https://pubchem.ncbi.nlm.nih.gov/compound/Desloratadine

[2] Mohammad, Y., Pragati Kumar, B., Srinivas, R., Rehana, S. K. (2010). New UV spectrophotometric method for the determination of desloratadine in bulk and tablet dosage forms. Journal of Chemical and Pharmaceutical Sciences, 3 (4), $241-243$.

[3] Validation of Analytical Procedures: Text and Methodology Q2R1 Current Step 4 version (2005). The International Conference on Harmonisation of Technical Requirements for Registration of Pharmaceuticals for Human Use. ICH Harmonised Tripartite Guideline, 17.

[4] Technical guide for the Elaboration of Monographs (2015). European Directorate for the Quality of Medicines \& HealthCare. Strasbourg: EDQM, 74.

[5] <1225> Validation of compendial procedures (2017). The United States Pharmacopeia 40. Rockville: The United States Pharmacopeial Convention, 1780-1787.

[6] Supplementary guidelines on good manufacturing practices: validation (2006). Technical Report Series. No. 937. Geneva: World Health Organization, 107-178.

[7] Koch, W. F., Hauck, W. W., de Mars, S. S., Williams, R. L. (2010). Measurement Science for Food and Drug Monographs: Toward a Global System. Pharmaceutical Research, 27 (7), 1203-1207. doi: http://doi.org/10.1007/s11095-010-0118-6

[8] Martin, G. P., Barnett, K., Burgess, C., Curry, P. D., Ermer, J., Gratzl, G. S. et. al. (2013). Lifecycle Management of Analytical Procedures: Method Development, Procedure Performance Qualification, and Procedure Performance Verification. Pharmacopeial Forum, 39 (5). Available at: https://www.uspnf.com/sites/default/files/usp_pdf/EN/USPNF/revisions/lifecycle_pdf.pdf

[9] Argentine, M., Barnett, K., Chatfield, M. et. al. (2017). Evaluating Progress in Analytical Quality by Design. Pharmaceutical Technology, 41 (4), 52-59.

[10] Magnusson, B., Örnemark, U. (Eds.) (2014). The Fitness for Purpose of Analytical Methods - A Laboratory Guide to Method Validation and Related Topics. Eurachem, 70.

[11] Bettencourt da Silva, R., Williams, A. (Eds.) (2015). Setting and Using Target Uncertainty in Chemical Measurement. Eurachem/CITAC, 30 . 
[12] Leontiev, D., Volovyk, N. (2016). Specificity of application of the uncertainty concept to the decision on compliance of medicines. Arhiv za farmaciju, 66, 207-208.

[13] The State Pharmacopoeia of Ukraine. Vol 2, Suppl. 4 (2020). Kharkiv: State Enterprise «Ukrainian Scientific Pharmacopoeial Centre for Quality of Medicines».

[14] Gryzodub, O., Leontiev, D., Denisenko, N., Podpruznikov, Y. (2004). Standardised procedure of validation of assay procedures by the method of standard. Farmacom, 3, 3-17.

[15] The State Pharmacopoeia of Ukraine. Vol 1, Suppl. 1 (2004). Kharkiv: State Enterprise «Ukrainian Scientific Pharmacopoeial Centre for Quality of Medicines».

[16] <1220> Analytical Procedure Lifecycle (2020). Pharmacopeial Forum, 46 (5). Available at: https://online.usppf.com/usppf

[17] Barnet, K., McGregor, P., Martin, G. P., et. al. (2017). Analytical Target Profile: Structure and Application Throughout the Analytical Lifecycle. Pharmacopeial Forum, 42 (5). Available at: http://www.usppf.com/pf/pub/index.html

[18] Takano, D. E. N., de Souza Reis, P. R., Singh, A. K., Lourenço, F. R. (2017). Estimation of uncertainty for measuring desloratadine in tablets formulation using UV spectrophotometry. Measurement, 101, 40-44. doi: http://doi.org/10.1016/j.measurement.2017.01.018

[19] Gryzodub, O. (2016). Standardised validation schemes for drug quality control procedures. Kharkiv: State Enterprise «Ukrainian Scientific Pharmacopoeial Center for Quality of Medicines», 396.

[20] <1200> Requirements for Compendial Validation (2014). Pharmacopeial Forum, 39 (6). Available at: http://www.usppf.com/ pf/pub/index.html 